Voix et Images

voixetimages

\title{
De la Nuit aux Confitures de coings : le poids des événements d'Octobre 1970
}

\section{Jacques Pelletier}

Volume 8, numéro 3, printemps 1983

Jacques Ferron

URI : https://id.erudit.org/iderudit/200397ar

DOI : https://doi.org/10.7202/200397ar

Aller au sommaire du numéro

Éditeur(s)

Université du Québec à Montréal

ISSN

0318-9201 (imprimé)

1705-933X (numérique)

Découvrir la revue

Citer cet article

Pelletier, J. (1983). De la Nuit aux Confitures de coings : le poids des événements d'Octobre 1970. Voix et Images, 8(3), 407-420. https://doi.org/10.7202/200397ar d'utilisation que vous pouvez consulter en ligne.

https://apropos.erudit.org/fr/usagers/politique-dutilisation/ 


\title{
De la Nuit aux Confitures de coings: le poids des événements d'Octobre 1970
}

\author{
par Jacques Pelletier, Université du Québec à Montréal
}

En 1965, la Nuit parait pour la première fois chez Parti pris. En 1972, sept ans plus tard, le même éditeur publie une "version entièrement nouvelle"1 de ce récit sous un nouveau titre: les Confitures de coings, version que l'auteur a mise au point l'année précédente et qu'il présente dans une lettre à Gérald Godin comme une "version corrigée" du texte de 1965.

Le sous-titre des Confitures de coings fait problème dans la mesure où il ne coincide pas parfaitement avec l'indication contenue dans la lettre à Gérald Godin"; entre une "version entièrement nouvelle" d'un texte et une "version corrigée" if y a une marge considérable: dans le premier cas il y a production d'un texte vraiment nouveau auquel le premier a pu servir de générateur - ce qui,me semble être le cas par exemple, dans l'oeuvre de Victor-Lévy Beaulieu, des Mémoires d'outre-tonneau pour Satan Be/humeur - alors que dans le second les modifications, même substantielles à l'occasion, n'affectent pas pour autant la signification d'ensemble du texte.

Qu'en est-il des productions de Ferron? Seule une lecture attentive, compara tive et analytique, des textes de 1965 et de 1972, pourra nous le dire, au-delà des indications explicites - mais extratextuelles - contenues dans le sous-titre de 1972 et dans les déclarations de l'auteur.

Par ailleurs, que la version de 1972 soit "entièrement nouvelle" ou plus modestement et simplement "corrigée", il me paraît intéressant de poser dans un second temps la question des raisons qui ont motivé les transformations opérées sur le texte de base: à quelles considérations stylistiques et/ou politiques leur étude nous renvoiet-elle? Et, pour annoncer tout de suite mes couleurs, il me semble que dans cette opé ration on ne pourra faire l'économie de l'étude des rapports du texte ferronnien à cet événement majeur de l'histoire contemporaine du Québec qu'a constitué la crise d'Octobre 1970.3

Dans la seconde version comme dans la première, on nous raconte une nuit dans la vie du héros, François Ménard, nuit décisive au terme de laquelle celui-ci retrouve son åme perdue des années plus tôt au début de l'âge adulte. L'action du récit - selon la procédure suggérée par Barthes (à la suite de Propp) - peut être très sommairement décrite comme suit: 
État initial: le héros, homme "moyen", banal, typique de la civilisation moderne, gérant de banque résidant en banlieue de Montréal, reçoit une nuit un appel téléphonique qui le reporte à son passé, oublié, enfoui dans la vie quotidienne, passé dont le moment déterminant a été, à l'aube de sa vie adulte, le reniement de ses convictions communistes et l'adhésion - tout au moins explicite, apparente, sinon en profondeur - aux valeurs dominantes.

État terminal: le héros, à la fin d'un parcours de nature initiatique vécu lors de cette fameuse nuit, recouvre son âme perdue et du coup cesse de parasiter celle de sa femme dont il a vécu durant des années: désormais redevenu un être authentique, il pourra reprendre le combat déserté à la fin de sa jeunesse.

Entre ces états (initial et terminal), le parcours, la "boîte noire" de Barthes comprenant les éléments suivants - je schématise ici énormément pour m'en tenir à l'essentiel - :

a) le départ à la suite du coup de téléphone de Frank; le héros se sent investi d'une mission: retrouver son âme;

b) la rencontre avec Frank à la Morgue, rue Saint-Vincent; le héros se remémore alors sa jeunesse: le séjour au sanatorium notamment, la rencontre avec Smédo, la conversion au communisme, l'engagement dans le Parti, la manifestation contre I'O.T.A.N. rue Saint-Laurent, l'arrestation et le reniement dont Frank sera l'artisan;

c) I'affrontement a l'Alcazar avec Frank sous forme du don d'un pot de confitures de coings empoisonnées; le héros se souvient à nouveau, cette fois de son passé plus lointain, l'enfance à Louiseville, dans Maskinongé dont Frank, son alter ego, est aussi originaire;

d) I'initiation amoureuse, dans un hôtel de passe de la rue Stanley, avec Barbara, petite négresse de Sydney, Nova Scotia - grâce à qui il retrouve pour une large part son âme;

e) le retour, d'abord à l'Alcazar, où le héros retrouve Frank mort, empoisonné, allongé les bras ballants sur une table de la boîte de nuit, puis à la maison de banlieue, où it retrouve Marguerite et sa vie routinière qu'il aborde cependant désormais sous un jour différent, étant redevenu d'une certaine façon le jeune homme idéaliste qu'il était naguère.

La transformation s'est donc effectuée sur le mode du voyage: a) dans l'espacetemps d'une nuit décisive, faisant tourner le destin; bl dans la redécouverte à l'inté rieur de cet espace-temps du moi profond remontant à l'enfance et à l'adolescence, moi recouvert durant la vie adulte par un masque voilant l'essentiel, à savoir, comme Ménard l'avait découvert au sanatorium, que "la réalité se dissimule derrière la réalité" et que le paisible banquier cache un "ex" et sans doute, après cette nuit initiatique, futur révolutionnaire. Ce voyage, ce parcours, on pourrait aussi bien l'illustrer par le schéma suivant ${ }^{5}$ : 


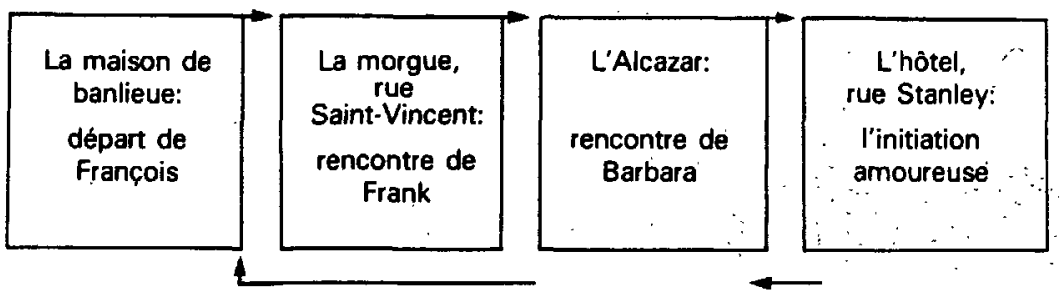

- Ce parcours, à la fois linéaire et circulaire, s'inscrit par ailleurs dans une structure plus englobante que l'on peut représenter ainsi:

le passe

le présent

le futur

1) Enfance à Maskinongé; études;

2) Maladie; conversion au communisme, décoùverte de la réalité;

3) Manifestation: procès et reniement

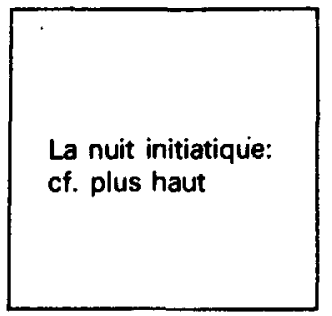

Au terme de la nuit: François transformé; ouverture sur l'avenir

Sur le plan de la structure et, par conséquent, de la signification d'ensemble du récit, il y a donc une parenté profonde - pour ne pas dire une parfaite identité - entre les deux versions de la Nuit, si bien que s'il y a des changements majeurs de la première à la seconde, ce ne sera pas à ce niveau central, déterminant qu'il faudra les repérer mais à celui des éléments porteurs de la structure: mode de représentation des personnages, déplacements d'accent en ce qui a trait à la(aux) thématique(s) du récit, utilisation différente des éléments référentiels etc. Mais d'ores et déjà on peut conclure, du moins provisoirement, que le texte publié en 1972 constitue sans doute plus une version "corrigée" qu'une "version entièrement nouvelle" du texte de 1965, ce qui ne préjuge en rien de l'importance (capitale ou secondaire) des transformations apérées sur le texte.

Diane Potvin dans une étude comparative rigoureuse ${ }^{6}$, a dénombré 950 unités de variance dans les Confitures de coings; ces unités de variance sont parfois d'ordre mineur - changement de mots, de signes de ponctuation, etc, - parfois d'ordre majeur - ajout ou suppression de paragraphes et même parfois de quelques pages altérant profondément le sens d'un passage - ; du point de vue qui m'intéresse, ce sont surtout les transformations de cet ordre qui $m$ 'importent, car elles sont de nature à mettre éventuellement en jeu la signification du texte à certains endroits stratégiques alors que les premières, si j'en juge par les exemples invoqués par Diane Potvin, obéissent à un pur souci de correction stylistique et sont donc, par conséquent, d'une portée très limitée. 
Première transformation significative: le titre qui, de la Nuit, devient les Confitures de coings avec précision en sous-titre qu'il s'agit d'"une version entièrement nouvelle de la Nuit'; à quoi on peut rattacher l'ajout de l'Appendice, aussi accompagné d'un sous-titre, "Le congédiement de Frank-Archibald Campbell": cet Appendice est extrêmement important, dans la mesure où il éclaire la genèse du texte et en propose une lecture fort révélatrice; on y reviendra plus loin. Deuxième changement à signaler au niveau du métatexte: la disparition dans les Confitures de l'extrait d'un poème de Duncan Campbell Scoth (sic), figurant en épigraphe de /a Nuit, et son remplacement par une dédicace à madame Elizabeth Bednarski.

Dans le texte méme, les modifications, quantitativement les plus importantes, portent sur le premier chapitre. A noter d'abord - il s'agit de la transformation la plus considérable et l'une des plus significative du récit - le développement sur trois pages dans les Confitures du premier paragraphe de la Nuit, et plus précisément de sa première phrase. Celle-ci, dans la version de 1965, s'énonçait comme suit: "Je n'ai jamais pensé que j'étais un imbécile; j'en avais quand même le salaire"7. Dans la version de 1972, elle est corrigée: "Je n'ai jamais pensé être un imbécile et personne ne me l'a dit; je ne l'aurais pas cru"B et prolongée, approfondie dans une longue méditation de trois pages sur la condition de solitude absolue dont tout homme est prisonnier: "On a beau faire, chacun reste soi, l'autre reste l'autre"9. "...on ne vit jamais, malgré qu'on en ait, qu'avec soi seul" 10 , ce sont là des échantillons d'un propos repris sous de multiples formes dans ce substantiel ajout.

La seconde transformation d'importance - sur le plan quantitatif tout au moins - apportée dans le premier chapitre concerne le personnage de Marguerite. Ici encore Ferron procède par ajout, développant sur quelques pages la description de Marguerite, qui tenait en trois paragraphes dans la version de 1965. Pour l'essentiel, toutefois, ce portrait prolonge plus qu'il ne modifie l'esquisse de 1965: l'épouse du héros-narrateur est toujours représenté comme une femme passive, bonne ménagère, sachant se contenter de peu et guère préoccupée par la poursuite du bonheur. La seule véritable modification concerne l'attitude du héros-narrateur à l'égard de son épouse: alors que dans la version de 1965 il n'était pas sûr d'être responsable de son malheur, il en paraît convaincu dans la version de 1972: "Un homme qui ne serait pas responsable du malheur de sa femme, écrit-il, n'est pas un homme"11. Cette seconde "correction" recoupe et complète en un sens la première, contribuant à accorder au récit un caractère plus intimiste que dans la version de 1965.

La troisième transformation opérée dans le premier chapitre a trait au personnage de Frank. Dans la Nuit, la représentation initiale de Frank est plutôt neutre alors que dans les Confitures elle est polémique. Dans le texte de 1965 en effet, Frank, à toutes fins pratiques, lorsqu'il est mis en scène pour la première fois, n'est pas décrit' alors que dans le texte de 1972 il est évoqué successivement comme un "prophète", un "géant", le "représentant des Rhodésiens montréalais? Le policier légiste?", un "pseudo-politicologue, une doublure peut-être du policier légiste"13, toutes images et surtout les dernières - qui le peignent çomme un personnage inquiétant, hostile. dont il faut se méfier et prendre le parti de le considérer comme un ennemi. Frank étant le second personnage ên importance du récit, ce changement est particulièrement significatif. 
Quatrième - et dernière - modification qui n'affecte pas un personnage, cette fois, mais la représentation d'un élément référentiel: le N.P.D. Dans la Nuit ce parti est décrit comme un "parti socialiste, le plus respectable, qui, tout en restant loin du gouvernement, lui fournissait les idées quand d'aventure il était réduit à l'extrémité d'en avoir"14. Bien entendu cette description comporte une part d'ironie car le hérosnarrateur précise bien qu'il ne s'est inscrit à ce parti que lorsqu'il eut atteint le statut de gérant de banque, établissant ainsi une sorte d'équation entre la respectabilité du parti "de gauche"et sa propre respectabilité personnelle de nouveau notable. Dans les Confitures, l'ironie fait place à la colère et c'est dans des termes vitrioliques que ce parti est dépeint: "je m'inscrivis au P.S.D., parti plus ou moins socialiste, plutôt moins que plus, issu de la C.C.F., qui allait devenir le N.P.D., et je n'étais pas loin de me faire l'effet, pauvre de moi, dans ce parti de tout repos, dans cette guimauve au fond écoeurante, dans cet attrape-nigaud pour les idéalistes et les gens de bonne volonté, dont on ne savait que faire, d'un extrémiste"15.

Dans les chapitres suivants, les transformations, a quelques exceptions près, seront généralement moins importantes sur le plan quantitatif et surtout moins significatives. C'est ainsi, à titre d'exemple, qu'un sergent de police au service des forces de répression lăchées contre les manifestants anti-O.T.A.N., baptisé Wagner dans la Nuit - sans doute "en l'honneur" du Ministre de la Justice de l'époque, partisan d'une politique musclé à l'égard des forces contestataires de la société québécoise - deviendra, dans les Confitures, le sergent Melançon, nom neutre n'évoquant aucun personnage officiel connu, substitution qui trouve sans doute ses raisons dans la relative éclipse de la scène politique de Wagner en 1972.

Plusieurs "corrections" sont de cette nature et donc peu pertinentes à mon propos. Aussi je me contenterai de relever rapidement celles qui me semblent comporter plus de conséquences. Je signale d'abord la présence, dans le texte de 1972, de Jean Drapeau (non nommé dans le texte de 1965) associé a Pax Plante et à son entreprise douteuse - selon le héros-narrateur - de moralisation publique: Plante est traité "d'énergumène moraliste" alors que Drapeau est qualifié $d$ " annonciateur des bains de sang" ${ }^{\prime \prime}$. Je signale ensuite l'amputation, dans les Confitures, d'une strophe d'un poème de Samuel Butler qui était présente dans la Nuit, suppression parmi d'autres qui, selon Guy Monette, traduirait une volonté de l'auteur de chasser l'occupant à tous les niveaux, de le poursuivre dans ses derniers - et moindres - replis", "soustraction" par ailleurs compensée par l'ajout d'un poème du "cousin Rodolphe." absent du texte de $1965^{18}$.

Plus importantes encore sont les modifications apportées à la représentation de Frank et du héros-narrateur. Dans un passage stratégique de la Nuit qui concerne les rapports entre les deux principaux protagonistes du récit, Frank est défini comme un Écossais alors que, dans le passage équivalent des Confitures, il est ramené à sa qualité génerique de Britannique' ${ }^{19}$. II s'agit là d'une modification de taille lorsqu'on sait que, pour Ferron, s'il y a possibilité d'entente pour les Québécois avec les Écossais, les Irlandais, les Gallois - nations dominées par l'empire britannique - il n'en va pas de même avec les Anglais, puissance impériale dominatrice ${ }^{20}$. Dans la même veine, le heros-narrateur décrira encore Frank dans les Confitures comme un "étranger amical. Dourtant mon ennemi" ${ }^{21}$, passage qui ne figurait pas dans la Nuit et qui 
accentue le caractère objectivement hostile du personnage de Frank. A cette "britannisation" de Frank, si l'on me passe ce néologisme, va correspondre un "enquébécoisement" encore plus prononcé du héros-narrateur devenant un symbole de la collectivité nationale dans un passage capital suivant immédiatement la reproduction d'un extrait du Gotha de Frank:

"Ces considérations distinguées et sans doute intelligentes me laissèrent indifférent. J'étais de nationalité québécoise, assurément, un peu comme je me serais nommé Ducharme ou Lachance, captif de mon origine, participant à un discours commencé avant moi, $y$ ajoutant mon mot, ma phrase, un point, c'est tout. Que pouvais-je faire de plus, surtout après cette nuit où je venais de renouer avec un temps perdu, la première personne d'un pluriel particulier, nous familial ou nous national, que m'importait, avec un temps qui ne m'était rien de plus qu'un espace, une ambiance, l'air qu'on respire, indispensable, certes, mais auquel on ne pense pas? Je ne revendiquais que le droit de $m$ 'isoler en moi-même et de $m^{\prime} y$ dissoudre en paix ${ }^{\prime 22}$.

Enfin, dernière modification majeure: la disparition du passage sur l'Effelquois. On sait qu'au retour à sa maison de banlieue le héros-narrateur de la Nuit rencontre un Effelquois en train d'effectuer des corrections à un poteau indicateur; Ménard lui fait un signe de complicité auquel l'autre finit par répondre, abolissant ainsi la distance entre les deux générations unies, tout compte fait, dans un même combat qu'elles mènent chacune à leur manière avec des armes différentes. Or ce passage, dont la signification politique ne saurait ètre sousestimée, n'a pas été repris dans les Confitures.

En somme les transformations les plus significatives du texte ont trait: 1. au titre, modifié, comme le dit l'auteur dans l'appendice, pour insister sur le poison, donc sur la nécessité de se débarrasser de Frank et de ce qu'il représente; 2 . au personnage de Ménard dont la."dimension intérieure" est accentuée d'un côté - ce qui implique du coup un traitement plus élaboré du personnage de Marguerite - de méme que, de manière un peu paradoxale, sa dimension collective est approfondie d'un autre côté; 3. au personnage de Frank présenté dans la seconde version comme un véritable ennemi et non plus comme un éventuel complice; 4, a la disparition du passage sur l'Effelquois.

Comment rendre compte de ces transformations, importantes dans la mesure où elles débordent le cadre de préoccupations d'ordre purement stylistique, mais néanmoins "secondaires" dans la mesure où elles n'affectent pas la structure de base du récit - dans la version de 1965 comme dans celle de 1972?

Les critiques de l'oeuvre de Ferron - notamment Diane Potvin et Pierre L'Hérault - ont fait état bien entendu de ces modifications et compris que les événements d'Octobre 1970 en étaient dans une large mesure responsables. Mais, en raison même de son approche - sémiologique - Diane Potvin, en pratique, a eu tendance à accorder une importance négligeable à ce facteur qu'elle évoque mais qu'elle n'analyse pas. Quant à Pierre L'Herault, il insiste surtout sur les motivations stylistiques ayant pu servir de fondements aux modifications et estime que certains changements - notamment la dispariton du passage sur l'Effelquois - trouvent leur raison d'être dans les textes ajoutés en 1972: la Créance et l'Appendice aux Confitures de coings $5^{23}$. Or il 
me semble qu'il faut aller plus loin et reconnaître que la crise d'Octobre 1970, loin d'être seulement une toile de fond liant plus ou moins láchement les deux versions de la Nuit, constitue le principal facteur explicatif des transformations opérées en 1972 sur le texte de 1965.

Ces événements, auxquels Ferron a participé activement, négociant à leur demande la reddition des frères Rose et de Francis Simard, ont une importance capitale dans I'histoire contemporaine du Québec pour plusieurs raisons dont, dans la perspective nationaliste qui est celle de l'auteur de la Nuit, les suivantes ${ }^{24}$ :

1. Les événements, à toutes fins pratiques, sonnent le glas du terrorisme au Québec dont la première vague remontait au printemps de 1963; tout se passe comme si les partisans de l'action violente réalisaient enfin: al que ce type d'intervention n'atteint pas les effets qui en étaient escomptés, soit la politisation progressive des masses sous l'influence d'actions exemplaires; b) que l'ennemi est imbattable sur le terrain militaire et qu'il est donc préférable de l'attaquer ailleurs;

2. Une partie de la gauche indépendantiste se rallie au P.Q. C'est le cas de Vallières accordant son appui tactique au P.Q. dans l'Urgence de choisir. C'est le cas également d'ex-animateurs de la revue Parti pris rejoignant les rangs de ce parti: Philippe Bernard, Gabriel Gagnon, Gérald Godin, etc. Le P.Q. consolide dónc son hégé monie sur le mouvement nationaliste;

3. En intervenant militairement, le pouvoir fédéral a indiqué au néo-nationalisme les limites à ne pas franchir; il n'acceptera pas la balkanisation du Canada. Cependant, de manière quelque peu paradoxale, la thèse péquiste de la souverainetéassociation sort renforcée de la crise, apparaissant comme une sorte de "troisième voie" entre un fédéralisme bloqué et l'indépendantisme pur et dur.

Sur les événements et leurs alentours, Ferron a beaucoup écrit, d'une part dans le cadre de chroniques d'actualité, publiées dans des journaux et des revues, qui ont été réunies par la suite dans les Escarmouches et d'autre part dans l'Appendice aux Confitures. De la lecture de ces textes il ressort que l'écrivain s'est intéressé essentiellement à la signification de la crise - dont il propose ce qu'il faut bien appeler une interprétation policière - et au rôle qu'y ont joué, à leur niveau et à leur manière, les anglophones du Québec traditionnellement reconnus comme des "libéraux".

\section{L'interprétation"policière" de Ferron repose sur les éléments suivants:}

1. Certains des felquistes de 1970 étaient des personnages douteux, des "gens masqués", si bien qu'on peut avancer l'hypothèse qu'ils étaient " $d$ 'accointance" avec la police ${ }^{25}$ soit comme complices et donc parties prenantes du complot, soit comme victimes manipulées par le pouvoir;

2. Cette manipulation a une longue histoire, le F.L.Q. ayant été, dès l'origine en 1963, sous la direction d'agents doubles, notamment d'origine belge, qui l'ont "fabriqué" et qui "restent actifs" encore aujourd'hui ${ }^{26}$;

3. Certains des membres du premier réseau felquiste en 1963 provenaient des Jeunesses communistes; or le parti communiste, réduit à presque rien après la deuxième guerre mondiale, servait essentiellement $d$ "'officine de police", de "poste 
d'observation"27 utilisé pour surveiller, contrôler et manipuler les éléments contes. tataires de la société québécoise; á l'appui de cet élément; Ferron invoque sa propre expérience d'ex-militant communiste;

4. La crise a été provoquée par les pouvoirs publics fédéraux pour mettre au pas les forces nationalistes du Québec et mème un soi-disant pacifiste comme Pearson, prix Nobel de la Paix, aurait été partie prenante d'un vaste complot visant à discré diter le P.Q. en I'associant au F.L.Q. ${ }^{28}$.

Si bièn que, l'un dans l'autre, "il y eut des conspirations en une seule, celle des terroristes qui par quelques exploits comptaient accélérer la marche de l'histoire. celle de la police et de l'establishment qui comptaient sur ces exploits pour imposer une fameuse terrorisation sociale ${ }^{\prime 29}$.

En somme, malgré sa sympathie pour certains terroristes, dont au premier chef les frères Rose auxquels il a consacré un texte émouvant dans le magazine McLean en $1971^{30}$, Ferron, dans l'ensemble, se méfie énormément du F.L.Q. et de tout ce qui lui ressemble de près ou de loin, dans la mesure où ces mouvements sont aisément manipulables comme l'a éloquemment démontré, selon lui, la crise d'Octobre 1970.

Dans cette entreprise de "terrorisation sociale", les intellectuels et les hommes politiques se sont révélés sous leur vrai jour en partisans ou adversaires de la manoeuvre d'intimidation. Les forces conservatrices, comme on pouvait s'y attendre, ont appelé et appuyé les mesures de répression. Mais les forces "progressistes", et singulièrement les démocrates anglo-saxons, libéraux "de gauche" ou "compagnons de route" du N.P.D., comment se sont-ils comportés devant l'événement? En défenseurs du Law and Order, en l'occurrence en apologistes de la Loi des mesures de guerre.

Ainsi, Frank Scott, se ralliant à Trudeau, après des "années de fourberie inconsciente" passées à la C.C.F. puis au N.P.D., admettait "à un åge avancé que, membre d'une minorité dominante, c'était outrager le sens commun que de se prétendre homme de gauche, et qu'à l'instar de tous ces Messieurs de la McGill University il était au fond un Rhodésien et qu'en faisant de son mieux il pouvait tout au plus atteindre le centre droit dans l'éventail des partis"31. Et dans l'Appendice, Ferron écrit que "délaissant ses frauduleuses utopies, jetant le masque, il s'est acoquiné sans vergogne à ce pauvre et misérable Pierre Trudeau, fils poisseux de Champlain et des Jésuites, à ce sacristain besogneux, tout renfrogné d'une religion révolue qu'est Gérard Pelletier..." ${ }^{23}$.

Scott, bien entendu, représente plus que l'individu Frank Scott: il symbolise - comme son double fictif, Frank Campbell - la communauté anglophone soi-disant progressiste de Montréal qui, dans ce moment de vérité qu'a constitué la crise, s'est révélée sous son vrai jour de minorité dominante tenant à ses privilèges et à son pouvoir.

La crise, en somme, pour Ferron, est le résultat d'un complot du pouvoir fédéral contre le nationalisme québécois, complot facilité par la participation aveugle, naive, de quelques jeunes idéalistes, terroristes trompés et manipulés, instruments d'une operation de terrorisation sociale de grande envergure dont fut victime une population aux abois. Elle a eu par ailleurs pour résultat de faire apparaitre sous leur jour véritable de soi-disant progressistes, faux alliés des "forces vives" de la nation québécoise: à ce titre, elle a servi de révélateur, permettant de délimiter très nettement le camp du 
peuple québécois et de ses amis et celui de ses adversaires.

Ceci posé, on comprend comment la crise, telle que perçue par l'écrivain, explique dans une très large mesure les modifications apportées dans le texte publié en 1972.

On a vu plus haut que les modifications majeures ont trait à quatre éléments: le titre, les personnages de Frank et du héros-narrateur, le passage sur l'Effelquois. Outre celles-ci, le texte de 1972 présente tout un ensemble de modifications qu'on peut considérer comme mineures et dont peut rendre compte, pour l'essentiel, une simple évocation de l'évolution de la conjoncture entre les deux périodes de rédaction. C'est ainsi, par exemple, que la disparition en épigraphe de l'extrait du poème de Duncan Campbell Scoth (sic) obeit, selon Guy Monette, à une volonté d'effacer dans l'oeuvre toute trace de l'occupant, du moins aux endroits stratégiques du récit. De mème, la description virulente du N.P.D. est alimentée par la prise de positions de certains de ses membres connus, dont Frank Scott se ralliant à Trudeau durant la crise d'Octobre; cependant, ce faisant, Ferron se montre très sévère - et disons-le assez injuste - pour un parti qui fut le seul, dans sa grande majorité, en votant contre le projet de loi décrétant les mesures de guerre, à refuser d' accorder au "fils poisseux de Champlain et des Jésuites" les pleins pouvoirs qu'il exigeait. Enfin la représentation de Jean Drapeau comme personnage sanguinaire trouve ses assises dans l'attitude fortement répressive manifestée par le maire de Montréal lors des événements.

Outre cette influence sur des modifications mineures apportées au texte de 1965, la crise (ou plus précisément la lecture qu'en fait Ferron) aura des conséquences sur des éléments-clefs de la Nuit.

A commencer par le titre. Dans l'Appendice, Ferron écrit que s'il prétère maintenant, suite aux événements, les Confitures de coings, c'est "pour insister sur le poison"33. Et effectivement ce changement de titre contribue à sa manière à ce qu'on pourrait appeler la "polémisation" - autre affreux néologisme! - du texte en mettant l'accent sur l'instrument matériel de la revanche du héros: le cadeau empoisonné qu'il fait à Frank. Le premier titre, la Nuit, ne contenait pas, du moins explicitement, cette connotation, insistant plutôt sur le parcours initiatique effectué par le héros, sur ses retrouvailles, lors d'une nuit décisive avec son moi authentique. Le second titre, lui, met en évidence la condition essentielle de cette reconquête là dimension collectivel d'une identité qui demeure problématique: l'élimination des ennemis, et d'abord des plus dangereux d'entre eux - les "libéraux" de McGill - car considérés à tort comme des alliés. La crise a indiqué, selon Ferron, l'urgence de cette tâche: "ll faut faire vite, derrière nous les ponts sont coupés, il n'y a plus de salut que dans l'occupation complète du pays..."34. C'est sur cet appel que se termine l'Appendice, invitant, dans la lutte qui va s'engager, à faire plus que jamais preuve de ruse - faute d'une force qu'on ne possède pas - à l'instar d'un Ménard utilisant avec efficacité l'arme à première vue dérisoire des confitures de coings.

Ce Ménard, on sait que Ferron a admis, toujours dans l'Appendice, qu'il était un avatar de lui-mème, victime d'une humiliation (le renoncement à son identité et à son authenticité) non pas tant personnelle que collective car lot de toute la commu- 
auté québécoise en situation de subordination face à une arrogante minorité dominante. On a vu, par ailleurs, que la dimension intérieure du personnage était approfondie dans les Confitures, ce qui peut paraître paradoxal compte tenu de la politisation accrue du texte mais qui ne l'est pas vraiment dans la mesure où les individus, chez Ferron, n'existent jamais à l'état d'atomes isolés mais appartiennent toujours à une communauté plus vaste - familiale, paroissiale, nationale, etc. - de laquelle ils héritent une dimension essentielle de leur être si bien que, séparés du groupe, ils sont perdus, comme l'illustrent éloquemment les exemples d'Aline Dupire dans la Lettre d'amour et de l'épouse de Baron dans les Roses sauvages. Cette intériorisation du personnage traduit, en outre, une préoccupation plus pressante, chez le Ferron du tournant des années 1970, qui se manifeste notamment dans des productions comme l'Amélanchier, les Roses sauvages, Du fond de mon arrière-cuisine, etc. qui témoignent de sa rencontre décisive avec la marginalité et la folie au Mont-Providence et à SaintJean-de-Dieu. Mais cette nouvelle préoccupation, dont le Ménard de 1971 est porteur, n'invalide pas la dimension collective du personnage, dont la "libération" ne pourra prendre son vrai sens qu'insérée dans celle de la communauté québécoise: il n'y a pas de salut individuel possible en situation d'oppression nationale et les événements de 1970 en ont à nouveau fait la preuve: d'où le caractère exemplaire - symbolique et collectif - de l'aventure de François Ménard, déjà affirmé en 1965, réaffirmé et renforcé en 1971.

Frank, l'alter ego de Ménard, son simulacre anglais, est, quant à lui, rejeté par I'auteur de l'Appendice qui le considère désormais comme un "ridicule épouvantail à corneilles, une manière d'imbécile presque aussi méprisable que ce Hugh MacLennan..." après l'avoir longtemps traité "avec révérence et une sorte d'amitié non seulement dans la Nuit mais aussi dans la Charrette et le Ciel de Québec "35. II n'est pas question ici d'analyser longuement les métamorphoses du personnage de Frank Archibald Campbell, ce qui pourrait constituer en soi l'objet d'une étude intéressante et instructive, aussi me contenterai-je de rappeler très brièvement le portrait qui en était donné dans les oeuvres antérieures à l'Appendice.

Dans la Nuit, on se souvient que Frank était représenté comme un ennemi certes mais avec lequel on pouvait discuter et éventuellement s'entendre. Le personnage, je l'ai rappelé, était d'ailleurs défini d'abord comme Écossais et non comme Anglais, membre actif de l'Empire. De plus, il possédait une sorte de double statut, à la fois d'adversaire réel du héros-narrateur et de double de celui-ci qui l'avait en quelque sorte intériorisé. Si bien que le combat était d'abord à livrer en soi, contre soi pour se transformer et non pas tant contre l'autre ${ }^{36}$ qui pouvait même devenir un allié.

Dans la Charrette (1968), Frank est décrit à nouveau essentiellement comme un Écossais de bonne volonté qui, non seulement veut comprendre le peuple québé cois mais lui appartenir, se réclamant dans un poème (p. 88-89) de cette nationalité. Cependant les Québécois demeurent sceptiques, ne disant ni oui ni non à cette demande de naturalisation. Le héros-narrateur du récit - nouvel avatar de l'écrivain, étant au surplus médecin comme celui-ci - a même tendance à trouver cette volonté d'enqué bécoisement de Campbell incongrue et assez absurde, bien que sympathique. Quant à Linda, personnage à travers lequel la question nationale est posée dans le roman. elle dira à Frank: "Surtout plus un mot de ta supposée nationalité québécoise! Ta déri- 
sion, garde-la pour les Ecossais" ${ }^{37}$. Le drame de Frank finalement, c'est qu'il n'a pas de véritable attaches: "Écossais, l'Écosse ne lui appartenait guère, ni le Québec, quoiqu'il en ait. Au fond c'était un homme détaché, plus porté au vagabondage qu'à la religion d'Etat" ${ }^{\prime 38}$. Sans ancrage historique précis, il est donc condamné à errer, comme le lui dira encore Linda, en "grand Don Quichotte inutile" 3 . Au total il apparait donc ici plus comme une victime que comme un ennemi et c'est à ce titre qu'il est dépeint avec sympathie.

Dans le Ciel de Québec (1969), le Frank mis en scène ne s'appelle plus Archibald Campbefl mais Frank Anacharsis Scot; cependant il est évident qu'il s'agit bien, sous ce nom nouveau, du même personnage. Dans la première partie de cette grande chronique des années 1930, Frank est représenté comme missionnaire dans les territoires du Nord-Ouest où, loin de précher la résignation, il enseigne la révolte aux Esquimaux. En raison de cet apostolat un peu particulier, on lui enlève sa mission et il rentre au Québec où il prend la décision, comme le Campbell de la Charrette, de s'enquébé coiser: "Dorénavant, dit-il, je me nommerai François-Anacharsis" ${ }^{40}$. Cette conversion semble tout à fait ridicule à son père, le bishop Dugald Scot qui estime que les Québé cois n'accepteront pas son fils "parce qu'il ont chacun leur petit privilège et qu'ils $y$ tiennent mordicus, à ce petit privilège, au point qu'ils auront peur de le perdre en vous voyant vous départir des prérogatives de votre naissance. Vous leur ferez peur tout simplement et ils ne sont pas des gens, que je sache, à vous en étre reconnaissants..." ${ }^{\prime \prime}$. Doute qui est également partagé par Chubby Power, politicien irlandais parfaitement assimilé, lui, et par l'abbé Surprenant - personnage qui sert souvent de porte-parole à Ferron comme on sait - qui confiera à Frank: "Laissez-moi seulement vous prévenir que quoi que vous fassiez, vous ne pourrez jamais vous enquébécoiser à fond; votre paradis perdu restera anglican; vos tartines risquent fort de vous laisser dans la bouche un arrière-goút de liberté anglaise" ${ }^{\prime 2}$. En dépit de ces conseils, Frank entreprend son enquébécoisement a) en se déniaisant dans un bordel de la rue Saint-Vallier; bl en se mettant au service des villageois des Chiquette, lieu dans le récit d'une possible renaissance québécoise. Le Ciel de Québec, donc, "finit bien" al par l'évocation de l'enfant sauveur, Rédempteur Fauché; bl par la "conversion" de Frank en Québécois. En cela il exprime le versant optimiste du nationalisme de Ferron, croyant en l'avenir d'un Québec ouvert, où il y place pour les Frank...

C'est cela qui est remis en question dans les Confitures et l'Appendice suite aux événements de 1970; d'où la description polémique de Frank comme "représentant des Rhodésiens montréalais", "policier légiste", "pseudo-politicologue", donc comme flic au service des forces de répression anti-québécoises. La crise de 1970 , à ce titre, a servi de révélateur, en forçant chacun à choisir son camp, en ne permettant plus les prises de position ambiguës, mettant ainsi crûment en lumière la duplicité de certains soi-disant amis du peuple québécois. Dans la mesure où le personnage de Frank les incarne dans les Confitures, on comprend le traitement que lui a réservé Ferron.

Dernière modification majeure apporté au texte: la disparition du passage sur l'Effelquois. La Nuit est publiée en 1965, donc, on s'en souviendra, au début du mouvement terroriste qui marque de façon spectaculaire l'éveil du néo-nationalisme qué 
bécois. À cette époque le F.L.Q. jouit d'une indéniable sympathie - voire de complicité - dans les milieux indépendantistes de gauche. Parti pris, fondée à l'automne 1963, se considère même d'une certaine façon comme un front intellectuel de libération du Québec. Et lorsque la revue critiquera en 1965-1966 la stratégie felquiste, ce sera sur fond de sympathie; entente sur les objectifs: nécessité d'une révolution polititique, sociale et économique au Québec; désaccord sur la stratégie ${ }^{43}$. "Compagnon de route" de Parti pris, militant indépendantiste, il était somme toúte normal que Ferron témoigne de sa compréhension - voire de son appui - au F.L.Q. C'est cela que manifeste le passage sur l'Effelquois dans la Nuit. Cette sympathie, on en trouvera aussi une expression dans le Salut de I'Irlande, roman publié en 1970 mais écrit avant les événements: le héros du livre, Connie Haffigan, un Irlandais, se convertit au felquisme tandis que ses frères aînés, eux, entrent dans la police... Si, en 1971, Ferron, reprenant la Nuit, fait disparaitre le passage sur l'Effelquois, c'est qu'il a compris que la stratégie felquiste est vouée à l'échec et que, de plus, elle se prête à toutes les manipulations comme les événements l'ont montré. Que Ferron ait eu ce pressentiment dès 1963, comme il l'affirme dans l'entrevue en tête de ce numéro, on peut bien sûr en douter - la Nuit et le Salut de l'Irlande inclinent plutót à penser le contraire mais ce n'est pas ce qui m'importe ici: ce qui est essentiel, c'est de constater qu'en 1971 un passage capital du texte de 1965 disparait et que cette modification est attribuable dans une très large mesure - sinon entièrement - à la lecture que l'écrivain a faite des événements d'Octobre 1970.

Alors les Confitures, "version entièrement nouvelle" ou plus simplement "corrigée" de la Nuit? Cette question. a un caractère un peu académique, avouons-le et on peut, à la limite, tout aussi justement répondre d'une façon ou de l'autre. Ce qui me paraît plus intéressant, c'est de souligner qu'il y a à la fois profonde continuité d'un texte à l'autre et rupture au niveau d'éléments importants, mais non stratégiques, du récit.

Continuité sur le plan de la structure et de la signification d'ensemble du texte. La matrice originelle de la Nuit - quête de l'identité personnelle et collective sur le mode d'un parcours initiatique - informe aussi les Confitures et trouve son enracinement dans la problématique nationaliste que Ferron s'est donnée à la fin des années 1950 et qui sert de toile de fond à toute son oeuvre depuis cette époque jusqu'a aujourd'hui.

Rupture au niveau d'éléments importants, mais non déterminants, du récit qui témoignent, à leur manière, de l'évolution de la conjoncture socio-politique et culturelle au Québec durant les années 1960 . Je n'insiste pas làdessus, ayant évoqué quelques-uns de ces changements au fil de mon texte; je rappelle seulement, très rapidement, qu'en dix ans les milieux nationalistes sont passés d'une conception "décolonisatrice" de la situation du Québec, inspirée par la lecture de Fanon, Memmi, Sartre, à une définition plus "fonctionnaliste" - le Québec comme élément à part entière du bloc américain sur qui pèse bien sûr une certaine oppression nationale - , ce qui s'est traduit sur le plan politique par une évolution de la revendication d'indépendance à celle de la souveraineté-association. Dans ce processus la crise d'Octobre 1970 a joué un rôle non négligeable et l'oeuvre de Ferron en rend compte à sa manière. 
1. Il s'agit du sous-titre de la nouvelle version; il n'est pas précisé si c'est ferron ou l'éditeur qui est à l'origine du sous-titre.

2. Lettre à Gérald Godin, en date du 27 juillet 1971, reproduite dans l'édition de 1977 des Confitures de coings, p. 8.

3. Ce texte, pour moi, s'inscrit donc aussi, à sa manière, dans le cadre d'une recherche plus. large sur les liens entre les événements d'Octobre 1970 et la littérature québécoise.

4. Op. cit., p. 46

5. Schéma mis au point par Monique Bólanger et Jacqueline Michaud-Landry à l'occasion d'un travail effectué dans le cadre d'un cours sur Ferron donné à l'hiver 1981 à l'Université du Québec à Rimouski.

6. Diane Potvin. "Ś́miologie de la variante chez Jacques Ferron Va Nuit et les Confitures de coings)", thèse de maitrise ès arts, Quebec, Université Laval, 1980, v., 128 pages.

7. La Nuit, Montréal, Parti pris, 1965, p. 9.

8. Les Confitures de coings, Montréal, Parti pris, 1977, p. 15.

9. Ibid., p. 16.

10. Ibid., p. 16.

11. Ibid., p. 21. Ce thème - il est intéressant de le noter - est repris et développé dans les Roses sauvages, texte également écrit et publié à cette époque.

12. La Nuit, p. 14.

13. Les Confitures de coings, p. 25-26.

14. La Nuit, p. 12.

15. Les Confitures de coings, p. 23. Je souligne.

16. Ibid., p. 57.

17. Cf. son article dans ce numéro.

18. Les Confitures de coings, p. 74-76.

19. Cf. la Nuit, p. 61 et les Confitures, p. 56.

20. Ca que démontrent, chacun à sa manière, le Ciel de Québec et le Salut de l'Irlande.

21. Les Confitures de coings, p. 87.

22. Ibid., p. 96.

23. Ct. les pages 241-242 de Jacques Ferron, cartographe de l'imaginaire, Montréal, P.U.M., 1980 (coll. "Lignes québécoises").

24. Je m'en tiens ici à l'essentiel tel que perçu dans une optique nationaliste; dans une perspective differente - socialiste par exemple - d'autres "leçons" de cette crise seraient à privilégier. Pour une analyse plus large de la signification de ces evénements, voir la première partie de mon article consacré a cette question dans la revue Conjoncture, Montréad, automne 1982.

25. Escarmouches, Montréal, Leméac, 1975, tome 1, p. 96. Ailleurs il écrira encore que "Cuba s'est empressé de prêter main forte à Ottawa en accueillant des Felquistes douteux, fabriqués sur mesure à même la postérité de Charlie Lanctôt" (p. 203).

26. Ibid., p. 224. Ferron soutient toujours ce point de vue en 1982, comme en témoigne l'entrevue qui ouvre ce numero.

27. Ibid., p. 97-98.

28. Opération qui, selon Ferron, sera aussi tentée contre le Parti Acadien: "L'apparition d'un F.L.A., ecrit-il en 1973 de retour d'un voyage à Moncton, ne m'a pas fait tellement plaisir: les Acadiens, me semble-t-il, ont déjà reçu leur part de violence. Qu'y apprendront-ils? Que F.L.A. et Sécurité s'articulent pour mieux sévir et réprimer" (lbid., p. 198).

29. Ibid., p. 176. Cette théorie du "complot" a été reprise et approfondie par Vallières dans /'Exécution de Pierre Laporte, ouvrage d'ailleurs dédicacé a Ferron. La "thérie" repose sur le postulat suivant: Washington, puissance impériale, est opposee a toute scission dans le bloc 
que forment les pays de l'Ouest. Pour éviter que cela ne se produise les dirigeants américains ont prévu des opérations économiques et militaires, dont le projet Camelot conçu pour le Chili sur le modèle d'un Revolt Project déjá expérimenté au Québec à partir de 1962. La crise d'Octobre 1970, dans cette perspective, a été fabriquée par le pouvoir fédéral let les services de police canadiens et américains) pour écraser une fois pour toutes le nationa lisme québécois et les felquistes, dans le meilleur des cas, ont été manipulés, dans le pire, ils ont été complices. A l'intérieur de ce cadre général d'analyse, les événements proprement dits sont ensuite reconstitués avec beaucoup d'imagination selon le journaliste Louis Fournier qui prépare, au moment où ces lignes sont écrites (juin 1982), un ouvrage historique sur la question qui prend à partie les interprétations du tandem Ferron-Vallières.

30. Repris dans Escarmouches, tome I, p. 103-118.

31. Ibid., p. 135.

32. Appendice aux Confitures de coings, p. 104.

33. Ibid., p. 105.

34. Ibid., p. 148.

35. Ibid., p. 105.

36. Ládessus se reporter au livre de Jean Bouthillette, le Canadien français et son double.

37. La Charrette, HMH, 1968, p. 123.

38. Ibid., p. 108.

39. Ibid., p. 115.

40. Le Ciel de Québec, Édition du jour, 1969, réédité par V.L.B. Ėditeur, 1979, p. 118.

41. Ibid., p. 259.

42. Ibid., p. $351-352$.

43. Cf., Jean-Marc Piotte, "Où allons-nous?", Parti pris, août-septembre 1965. 